\title{
Orientation Fields Filtering by Derivates of a Gaussian
}

\author{
Josef Bigun $^{1}$, Tomas Bigun ${ }^{2}$, and Kenneth Nilsson ${ }^{1}$ \\ 1 Halmstad University, SE-301 18, Halmstad, Sweden \\ 2 TietoEnator ArosTech, Storg. 3, SE-582 23 Linkping, Sweden
}

\begin{abstract}
We suggest a set of complex differential operators, symmetry derivatives, that can be used for matching and pattern recognition. We present results on the invariance properties of these. These show that all orders of symmetry derivatives of Gaussians yield a remarkable invariance : they are obtained by replacing the original differential polynomial with the same polynomial but using ordinary scalars. Moreover, these functions are closed under convolution and they are invariant to the Fourier transform. The revealed properties have practical consequences for local orientation based feature extraction. This is shown by two applications: i) tracking markers in vehicle tests ii) alignment of fingerprints.
\end{abstract}

\section{Introduction}

We present invariance properties of certain complex derivatives, symmetry derivatives, w.r.t. gaussians and show their applications. By means of these results, the structure tensor presented in $[1,2]$, which constitute a front end processing to produce orientation fields, e.g. for texture and fingerprint processing [3], as well as corner points, $[4,5]$, in an increasing number of applications, can be extended to model and match patterns that are more intricate than lines and edges. The applications we show consist in i) cross marker tracking in vehicle crash tests, ii) fingerprint alignment. From method point of view both applications are realized as orientation fields filtering. The contribution of the theorems to practice has been robust pattern recognition with predetermined number of arithmetic operations that were implemented via 1-D correlates.

The "angular" encoding representing the orientation fields of parabola and spiral like shapes were first proposed by [6] and [7]. Also, polynomial approximations of orientation filtering have recently been studied by [8] albeit the properties of the Gaussian derivatives were not studied.

The popularity of Gaussians as filters, is due to their valuable properties including: i) directional isotropy, i.e. in polar coordinates they depend on radius only, ii) separability in $x$ and $y$ coordinates, and iii) simultaneous concentration in the spatial and the frequency domain, e.g. [9-12]. In particular, efficient orientation analysis [1-3], and singularity points detection schemes $[4,5]$ have been utilizing Gaussians as interpolators and window functions.

We present the results as theorems and lemmas, the proofs of which are given in [13]. 


\section{Symmetry derivatives and the structure tensor}

Definitions We define the first symmetry derivative as the complex operator

$$
D_{x}+i D_{y}=\frac{\partial}{\partial x}+i \frac{\partial}{\partial y}
$$

which resembles the ordinary gradient in $2-\mathrm{D}$. When it is applied to a scalar function $f(x, y)$, the result is a complex field instead of a vector field. Consequently, the important difference is that it is possible to take the (positive integer or zero) powers of the symmetry derivative e.g.

$$
\begin{aligned}
& \left(D_{x}+i D_{y}\right)^{2}=\left(D_{x}^{2}-D_{y}^{2}\right)+i\left(2 D_{x} D_{y}\right) \\
& \left(D_{x}+i D_{y}\right)^{3}=\left(D_{x}^{3}-3 D_{x} D_{y}^{2}\right)+i\left(3 D_{x}^{2} D_{y}-D_{y}^{3}\right)
\end{aligned}
$$

The operator, $\left(D_{x}+i D_{y}\right)^{n}$ will be defined as the $n$ 'th symmetry derivative .

In an analogous manner, we define the first conjugate symmetry derivative as $D_{x}-i D_{y}=\frac{\partial}{\partial x}-i \frac{\partial}{\partial y}$ and the nth conjugate symmetry derivative as $\left(D_{x}-i D_{y}\right)^{n}$. We will however, only dwell on the properties of the symmetry derivatives. The extension of our results to conjugate symmetry derivatives are straight forward.

Sampled functions are denoted as $f_{k}$ i.e. $f_{k}=f\left(x_{k}, y_{k}\right)$. A capital letter represents the Fourier transformed function i.e. the Fourier transform of $f(x, y)$ is represented by $F\left(\omega_{x}, \omega_{y}\right)$. The notion power spectrum (of $f$ ) refers to $|F|^{2}$.

We apply the $p$ 'th symmetry derivative to the Gaussian and define the function $\mu^{\left\{p, \sigma^{2}\right\}}$ as

$$
\mu^{\left\{p, \sigma^{2}\right\}}(x, y)=\left(D_{x}+i D_{y}\right)^{p} \frac{1}{2 \pi \sigma^{2}} e^{-\frac{x^{2}+y^{2}}{2 \sigma^{2}}}
$$

with $\mu^{\left\{0, \sigma^{2}\right\}}$ being the ordinary Gaussian.

Theorem 1. The differential operator $D_{x}+i D_{y}$ and the scalar $\frac{-1}{\sigma^{2}}(x+i y)$ operate on a Gaussian in an identical manner:

$$
\left(D_{x}+i D_{y}\right)^{p} \mu^{\left\{0, \sigma^{2}\right\}}=\left(\frac{-1}{\sigma^{2}}\right)^{p}(x+i y)^{p} \mu^{\left\{0, \sigma^{2}\right\}}
$$

The theorem reveals an invariance property of the Gaussians w.r.t. symmetry derivatives. In comparison, the Laplacian of a Gaussian $\left(D_{x}^{2}+D_{y}^{2}\right) \mu^{\left\{0, \sigma^{2}\right\}}=$ $\left(-2 / \sigma^{2}+\left(x^{2}+y^{2}\right) / \sigma^{4}\right) \mu^{\left\{0, \sigma^{2}\right\}}$, a second order derivative too, can not be obtained by substituting $x y$ with $D_{x}, D_{y}$. As the example hints, the result of higher order derivatives will not automatically resemble the polynomial form of the derivation operators. Yet, such a form invariance is revealed by the theorem for any order of symmetry derivatives. Also, By using the linearity of derivation, the theorem can be generalized to an arbitrary polynomial.

Lemma 1. Let the polynomial $Q$ be defined as $Q(q)=\sum_{n=0}^{N-1} a_{n} q^{n}$. Then

$$
Q\left(D_{x}+i D_{y}\right) \mu^{\left\{0, \sigma^{2}\right\}}(x, y)=Q\left(\frac{-1}{\sigma^{2}}(x+i y)\right) \mu^{\left\{0, \sigma^{2}\right\}}(x, y)
$$


Theorem 2. The symmetry derivatives of Gaussians are Fourier transformed on themselves i.e.

$$
\mathcal{F}\left[\mu^{\left\{p, \sigma^{2}\right\}}\right]=\iint \mu^{\left\{p, \sigma^{2}\right\}}(x, y) e^{-i \omega_{x} x-i \omega_{y} y} d x d y=2 \pi \sigma^{2}\left(\frac{-i}{\sigma^{2}}\right)^{p} \mu^{\left\{p, \frac{1}{\sigma^{2}}\right\}}\left(\omega_{x}, \omega_{y}\right)(7)
$$

When constructing rotation invariant filters, [14] observed that the (symmetry) order $n$ in the functions, $h(r) \exp (i n \varphi)$ is preserved under the Fourier transform, however, without guidance on the choice of $h$ for full invariance. Likewise, the phase angles of the steerable filters, [11,15], are the same as those of $\mu^{\left\{p, \sigma^{2}\right\}}(x, y)$, however, without that the invariance properties are explicited.

Theorem 3. The symmetry derivatives of Gaussians are closed under the convolution operator so that the order and the variance parameters add under convolution.

$$
\mu^{\left\{p_{1}, \sigma_{1}^{2}\right\}} * \mu^{\left\{p_{2}, \sigma_{2}^{2}\right\}}=\mu^{\left\{p_{1}+p_{2}, \sigma_{1}^{2}+\sigma_{2}^{2}\right\}}
$$

The scalar $I_{p q}(\beta)=\iint(x+i y)^{p}(x-i y)^{q} \beta(x, y) d x d y$ with $p$ and $q$ being non-negative integers, is the complex moment $p, q$ of the function $\beta$. The order number and the symmetry number of a complex moment refer to $p+q$ and $p-q$ respectively. Consider only the second order complex moments of the power spectrum $I_{p q}\left(|F|^{2}\right)$ and the structure tensor $S,[1,2]$ :

$I_{p q}\left(|F|^{2}\right)=\iint\left(\omega_{x}+i \omega_{y}\right)^{p}\left(\omega_{x}-i \omega_{y}\right)^{q}\left|F\left(\omega_{x}, \omega_{y}\right)\right|^{2} d \omega_{x} d \omega_{y} \quad S=\iint \nabla f \nabla^{t} f d x d y$

with $I_{20}\left(|F|^{2}\right)$, complex, $I_{11}\left(|F|^{2}\right)$, real, and $S$ being a real $2 \times 2$ matrix. The two scalars $\left(I_{20}, I_{11}\right)^{t}$ and the structure tensor $S$ are fully equivalent. This is generalized in the next theorem.

\section{$3 \quad$ Pattern modelling and matching}

With few correlations (preferably 1-D), can sophisticated patterns and their directions be found? The next theorem attempts to answer this question.

Theorem 4 (Generalized linear symmetry). There is a structure tensor also in harmonic coordinates ${ }^{3}$. In particular, the second order complex moments determining the minimum inertia axis of the power spectrum, $\left|F\left(\omega_{\xi}, \omega_{\eta}\right)\right|^{2}$, can be obtained in the (Cartesian) spatial domain as:

$$
\begin{aligned}
I_{20} & =\iint\left(\left(D_{\xi}+i D_{\eta}\right) f\right)^{2} d \xi d \eta \\
& =\iint e^{i \arg \left(\left(D_{x}-i D_{y}\right) \xi\right)^{2}}\left[\left(D_{x}+i D_{y}\right) f\right]^{2} d x d y=\left(\lambda_{\max }-\lambda_{\min }\right) e^{i 2 \varphi_{\min }} \\
I_{11} & =\iint\left|\left(D_{\xi}+i D_{\eta}\right) f\right|^{2} d \xi d \eta=\iint\left|\left(D_{x}+i D_{y}\right) f\right|^{2} d x d y=\lambda_{\max }+\lambda_{\min }
\end{aligned}
$$

${ }^{3}$ A coordinate pair $\xi(x, y), \eta(x, y)$ is harmonic iff $D_{x} \xi=D_{y} \eta$ and $D_{y} \xi=-D_{x} \eta$, i.e. the curves $\xi(x, y)=\xi_{0}$ and $\eta(x, y)=\eta_{0}$ are perpendicular. 
The quantities $\lambda_{\min }, \varphi_{\min }$ and $\lambda_{\max }$ are, respectively, the minimum inertia, the direction of the minimum inertia axis, and the maximum inertia of the power spectrum of the harmonic coordinates, $\left|F\left(\omega_{\xi}, \omega_{\eta}\right)\right|^{2}$

Central to generalized structure tensor is the harmonic deformation function pair $x=\xi(x, y)$ and $y=\eta(x, y)$ which creates a new coordinate pair of curves to represent the points of the 2-D plane. Any analytic function $g(z)$ generates a harmonic pair via $\xi=\Re[g]$ and $\eta=\Im[g]$, representing the real and the imaginary parts of $g$. Monomials offer attractive computation schemes, as suggested next.

Lemma 2. Consider the analytic function $g(z)$ with $\frac{d g}{d z}=z^{\frac{n}{2}}$ and $n$ is any integer, $0, \pm 1, \pm 2, \cdots$. Then the discrete filter $\mu_{k}^{\left\{n, \sigma_{2}^{2}\right\}}$ is a detector for patterns generated by the curves $a \Re[g(z)]+b \Im[g(z)]=$ constant provided that i) a Gaussian is assumed to be the interpolator and ii) the magnitude of a symmetry derivative acts as a window function. The discrete scheme

$$
\begin{aligned}
& I_{20}\left(\left|F\left(\omega_{\xi}, \omega_{\eta}\right)\right|^{2}\right)=C \mu_{k}^{\left\{n, \sigma_{2}^{2}\right\}} *\left(\mu_{k}^{\left\{1, \sigma_{1}^{2}\right\}} * f_{k}\right)^{2} \\
& I_{11}\left(\left|F\left(\omega_{\xi}, \omega_{\eta}\right)\right|^{2}\right)=C\left|\mu_{k}^{\left\{n, \sigma_{2}^{2}\right\}}\right| *\left|\mu_{k}^{\left\{1, \sigma_{1}^{2}\right\}} * f_{k}\right|^{2}
\end{aligned}
$$

with $C=2 \pi^{2} e \sigma_{1}^{2} \sigma_{2}^{4}$ and $0 \leq n$ estimates the orientation parameter $\tan ^{-1}(a, b)$ as well as the error via $I_{20}$ and $I_{11}$ according to Theorem 4 . For $n<0$ the above scheme applies except that $\left(\mu^{\left\{-n, \sigma_{2}^{2}\right\}}\right)^{*}$ replaces $\mu^{\left\{n, \sigma_{2}^{2}\right\}}$

It should be noted that in the proof of the Lemma all previous results are utilized. To view the detectable patterns, we integrate $\frac{d g}{d z}=z^{\frac{n}{2}}$ and obtain

$$
g(z)= \begin{cases}\frac{1}{\frac{n}{2}+1} z^{\frac{n}{2}+1}, & \text { if } n \neq-2 \\ \log (z), & \text { if } n=-2 .\end{cases}
$$

Such patterns are shown in Figure 1 by displaying $s(t)=\cos (a \xi+b \eta)$. Lemma 2 estimates $\varphi=\tan ^{-1}(a, b)$ via the argument of $I_{20}$ regardless of $s$. In Figure $1 \mathrm{i}$ ), this angle is fixed to $\varphi=\frac{\pi}{4}$ and $n$ is varied between -4 and 3. Each $n$ represents a separate pattern class. By changing $\varphi$ and keeping $n$ fixed, the parameter pair $(a, b)$ is rotated to $\left(a^{\prime}, b^{\prime}\right)$. For $n \neq-2$, this results in rotating the iso-curves as displayed by Figure 1 ii) for increasing values of $\varphi$. When $n=-2$, the iso-curves are given by $g(z)=\log (|z|)+i \arg (z)$, which represents the only case when a change of the ratio between $a$ and $b$ does not result in a rotation of the image pattern but instead results in a bending of the the iso-curves, i.e. the spirals become 'tighter' or 'looser' until reaching the limit patterns, circles and radial patterns.

\section{Applications and experimental results}

\subsection{Symmetry tracker}

In vehicle crash tests, the test event is filmed with a high speed camera in order to quantify the impact of various parameters on human safety, by tracking markers 
i)

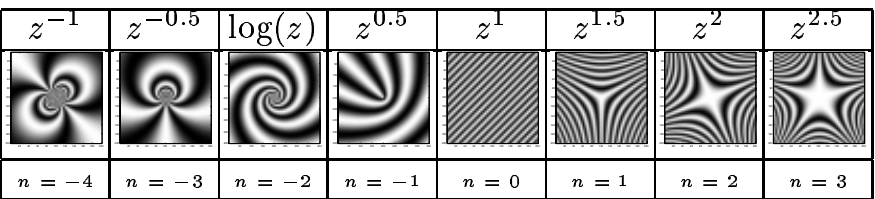

iii)

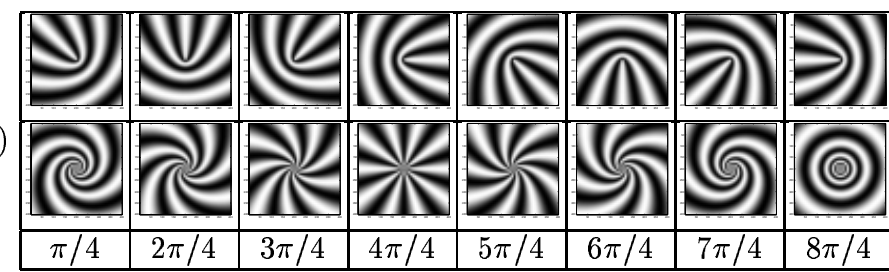

iv)

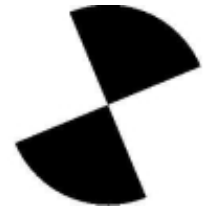

v)

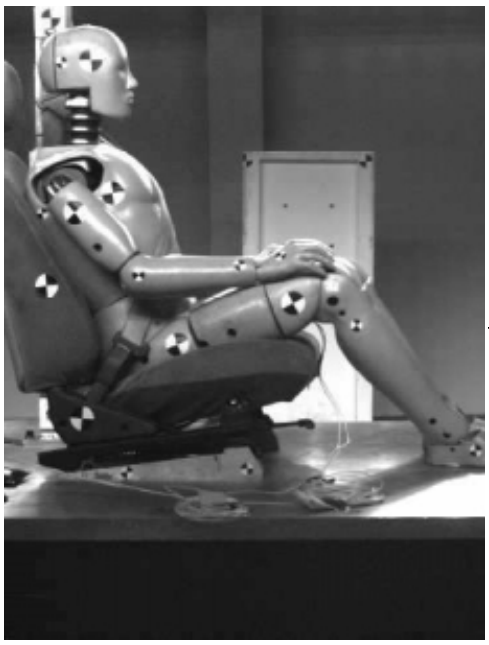

nusbowPaintt1 [x]

vii)

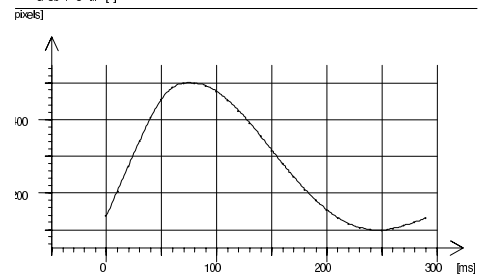

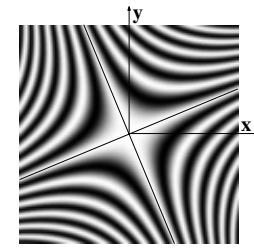

vi)

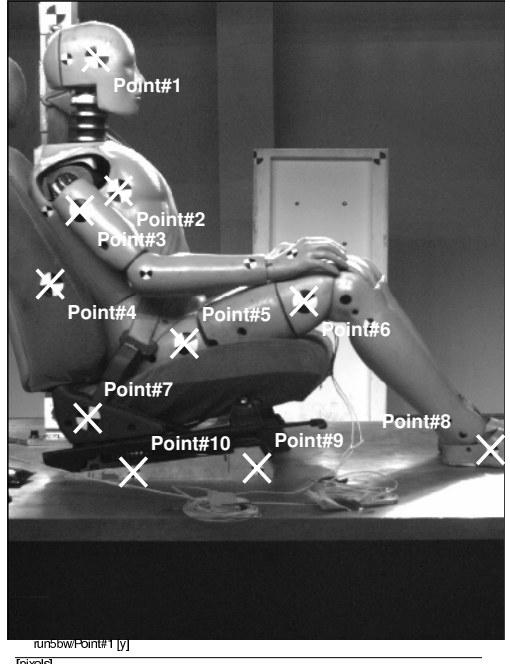

viii)

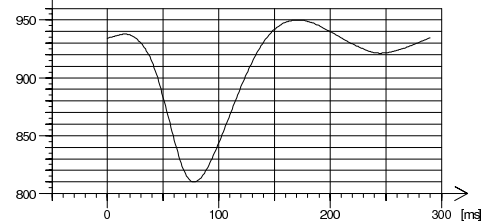

Fig. 1. i) On top, the harmonic functions (14) with the corresponding patterns, the (detection) filters as well as the symmetry orders are shown. ii) At bottom, the patterns generated by changing $\varphi$ is shown for $n=-1$ and $n=-2$ respectively. iii) The ideal model with $\varphi=\frac{\pi}{8}$. iv) The hyperbolic cross with $\varphi=\frac{\pi}{8}$. v) The first frame of an image sequence vi) The identified crosses, vii) and viii) The $\mathrm{x}$ and $\mathrm{y}$ coordinates versus time for Point \#1. 
including the "cross", see Figure $1 \mathrm{v}$ ). The cross tracking has to be robust i.e. the markers should not be lost from frame to frame even under severe light conditions, e.g. imperfect flash synchronizations.

The markers will be detected by applying Lemma 2 with $z^{\left(\frac{n}{2}+1\right)}$ and $n=2$. This generates not only patterns similar to crosses but also the ideal cross model when the definition $s(t)=\chi(t)$ where $\chi(t)$ is the step function (which is 1 for positive $t$, and 0 otherwise), is utilized, Figure 1 iii). As Lemma 2 suggests, the choice of $s$ itself, see Figure 1 iv) for another choice, has no-influence on the estimated $\varphi$. We use the rotation invariant measure (see Theorem 4) $C_{r}=$ $\left(\lambda_{\max }-\lambda_{\min }\right) /\left(\lambda_{\max }+\lambda_{\min }\right)=\left|I_{20}\right| / I_{11} \leq 1$ which compares the minimum error with the maximum error when finding the position of a marker. It attains its maximum iff $\lambda_{\min }=0$. The points having large certainties are candidate positions of markers. We state the algorithm that we used to track the markers as follows.

Algorithm: All steps are carried out in a region of interest, the search window. 1. Compute the complex image $h=\left(f_{x}+i f_{y}\right)^{2}$ by separable 1-D convolutions, $\mu^{\left\{1, \sigma_{1}\right\}} * f$, and pixel-wise complex squaring, 2. Compute the the complex image $I_{20}$, (12), by convolving the complex image $h$ with the complex filter of $\mu^{\left\{n, \sigma_{1}^{2}+\sigma_{2}^{2}\right\}}$, using separable convolutions, 3. Compute the real image $I_{11}$ by convolving the magnitude of the complex image of Step 1, with the magnitude of the filter in Step 2 via separable convolutions, (13), 4. Compute the certainty image, $C_{r}$, by pixel-wise division of the images, $I_{20}$ and $I_{11}$, obtained previously, 5. Compute the maximum of $C_{r}$ in the search window to obtain the position of the marker.

Evidently $n=2$ is utilized in Step 2. All steps are applied to all search windows and the maximum $C_{r}$ in a search window was identified. Initially (in frame 1), the search windows, containing one marker each, are found automatically. The size of the filters were 9x9 in Step 1, and a 30x30 in Step 2 and 3.

Tracking results and comparisons The algorithm has been tested by a number of crash-test experts. The robustness, few manual interventions, and fast executions are important issues. The cross-marker should not be lost from frame to frame, i.e. the found positions must be accurate. Figure 1 vi) shows a typical frame of the marker tracking process, superimposed to the original frame in iii). Also the $x$ and $y$ coordinates of one marker (\#1) in pixels versus time (frame no.), are shown in Figure 1. The graphs appear continuous which indicates that the "head" has not been lost and that the accuracy in the position is reliably quantified.

100 cross markers coming from several crash tests were utilized to test the performance. The symmetry tracker could track all but 5 markers. The used sequences included very long image sequences (in the order of thousands of frames). The algorithm lost the track of a marker when the contrast level of a cross was extremely poor e.g. a strong specular reflection.

An alternative method that was tested was correlation using an image of a typical cross marker as a template. The position accuracy was inferior to that of the symmetry tracker when the rotation was considerable between two frames. 
This is explainable because the correlation is minimum ( 0 , instead of maximum) when a cross rotates $\pi / 2$. On the other side using an iterative approach to correlation, e.g. [16], was not permissible because of hardware and time restrictions requiring i) the number of the iterations be known ii) numerous manual definitions of the search windows of the crosses in the initial frame since there are typically many markers that translate and rotate independently in the images. The method has lost the marker during tracking in 46 cases of 100 .

\subsection{Fingerprint alignment}

In biometrics, the alignment of two fingerprints without extraction of minutiae, typically an end of a line, has recently gained increased interest. Besides improved accuracy, this eliminates the costly combinatorial match of minutiae. We suggest to identify two standard landmarks: Core and Delta, see Figure 2 i). These are modeled and detected by a scheme based on Lemma 2 . It is similar to the are presented previously. Two analytic functions $z^{\frac{1}{2}}$, i.e. $n=-1$, and $z^{\frac{3}{2}}$, i.e. $n=1$ were used to model "Core" and "Delta", see Figure 1.

Algorithm: 1. Obtain the square of the derivatives via a convolution with $a 5 \times 5$ separable filter and complex squaring: $h_{k}=\left(\mu_{k}^{\left\{1, \sigma_{1}\right\}} * f_{k}\right)^{2}$. Then build a Gaussian octave pyramid of $h_{k}$ image (level 0 corresponds to the original size) up to level 3. The pyramid is built to improve the signal to noise ratio. 2. Convolve the highest level of the pyramid with the filters $\mu^{*\{1,1.5\}}$ for Core detection, and $\mu^{\{1,1.5\}}$ for Delta detection, (both $\left.9 \times 9\right)$ to obtain $I_{20}$ for each landmark type. 3. At the top of the pyramid and for each landmark, compute the real image $I_{11}$ by convolving the magnitude of the at Step 1, with the magnitude of the filter at Step 2 via (13), 4. At the top of the pyramid and for each landmark, compute the certainty image, $C_{r}$, by pixel-wise division of the images, $I_{20}$ and $I_{11}$, obtained in the previous two steps, 5. At the top of the pyramid and for each landmark type, compute the maximum of $C_{r}$ to obtain rough positions of the landmarks. Postprocessing Once the landmark positions and orientations were estimated at the top of the pyramid, the position parameters were fine-tuned by projection to a lower level. In that level, by carrying out computations analogous to those in steps 2-5 of the Algorithm, but applied in a $13 \times 13$ window centered around the position to be fine-tuned, the maximum $C_{r}$ s were found to update the positions of the two landmarks. The process was repeated until level 0 , where the final positions of the landmarks was found, was reached. The same procedure was applied to the second fingerprint image to be aligned. The translation between the two images is evidently obtained by the difference of the positions of the corresponding landmarks. The rotation parameter was obtained via the arguments of the complex scalars, $I_{20}$, at the positions. This was done by subtracting the thus obtained two arguments of $I_{20}$. If both of the landmarks were detected with certainties above 0.5 , only the translation and the orientation of the landmark having the maximum certainty, was retained.

Alignment results and comparisons

We report results on the FVC2000 fingerprints database, [17]. The FVC2000 contains a total of 800 fingerprints, many having a poor quality since they are 
i)

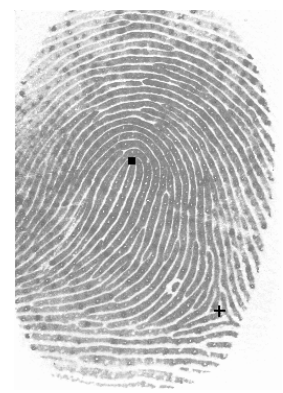

ii)

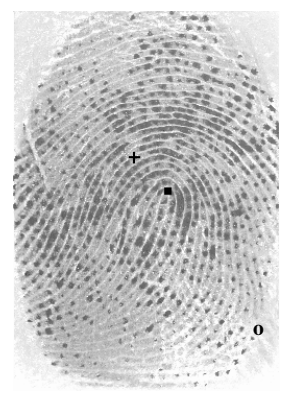

iii)

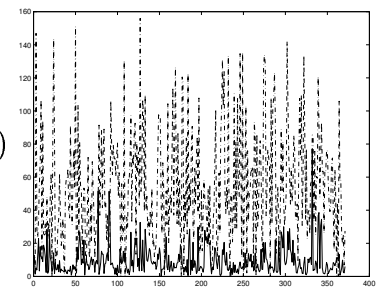

Fig. 2. The "+" and the "" represent the Delta and Core (parabola like) points that have the largest certainties. i) The certainties are 0.84 (Core) and 0.64 (Delta), ii) the certainties are 0.73 (Core) and 0.22 (Delta). The certainty at the Delta point marked with "O" is 0.10 . iii) The registration errors in pixels. The solid and dashed graphs correspond to the symmetry tracker and to the model based motion tracker, respectively.

captured using a low cost capacitive sensor from 100 persons, e.g. see Figure 2 for two fingerprints of the same finger. The Delta in Figure 2 ii), is an example of a difficult singularity point to identify even for human observers. It also shows the positions of the maximum certainties corresponding to Core and Delta as obtained by our approach. The system failed to assign the highest certainty to the correct position in Figure 2 ii), and suggested another (false) point. However, despite being maximum, the certainty for the (false) point being a true Delta point was low, 0.22. The false as well as the true point are below the used threshold of 0.5 so that this point was rejected alltogether by the system and only the point marked with "ם", has been retained to estimate the position and orientation parameters of the fingerprint.

The fingerprints of FVC2000 for a person have been translated and rotated with the computed parameters by our method towards one of his fingerprints. For each finger in the test image a minutia point other than the automatically found landmark (Core, Delta) was manually identified in the reference as well as in the test images to quantify the registration errors. In Figure 2 iii), these errors at manually found minutiae positions (our method in solid, and another method see below, in dashed,) are plotted. The horizontal axis is the index of the images.

We have also tested another method which employs (affine) model motion estimation and robust statistics to find the alignment parameters between two image, similar to [16] but with the M-estimator as regressor. Other tested regressors (LS, LMS, and LTS) were worse and are omitted here. The dashed graph of Figure 2 iii) corresponds to the errors of the model-based motion tracker. It shows that in nearly all images the alignment errors were significantly lower with the symmetry tracker. 


\section{Conclusion}

We have suggested the complex symmetry derivative operator. In 4 theorems and 2 lemmas we have revealed its properties and shown that these are of practical importance to 2 -D pattern recognition applications. In particular, we have presented experimental results when i) tracking crosses in long image sequences ii) aligning fingerprint images, using these results. These encourage the utilization of the symmetry derivatives in other contexts, e.g. texture feature extraction, image compression.

\section{References}

1. Bigun, J., Granlund, G.H.: Optimal orientation detection of linear symmetry. In: First International Conference on Computer Vision, ICCV (London), Washington, DC., IEEE Computer Society Press (1987) 433-438

2. Kass, M., Witkin, A.: Analyzing oriented patterns. Computer Vision, Graphics, and Image Processing 37 (1987) 362-385

3. Rao, A.R.: A taxonomy for texture description and identification. Springer (1990)

4. Forstner, W., Gulch, E.: A fast operator for detection and precise location of distinct points, corners and centres of circular features. In: intercommission conference on fast processing of photogrammetric data, interlaken. (1987) 281-305

5. Harris, C., Stephens, M.: A combined corner and edge detector. In: Proceedings of the 4'th Alvey Vision Conference, September. (1988) 147-151

6. Knutsson, H., Hedlund, M., Granlund, G.H.: Apparatus for determining the degree of consistency of a feature in a region of an image that is divided into discrete picture elements. In: US. patent, 4.747.152. (1988)

7. Bigun, J.: Recognition of local symmetries in gray value images by harmonic functions. In: Ninth International Conference on Pattern Recognition, Rome, IEEE Computer Society Press (1988) 345-347

8. Johansson, B.: multiscale curvature detection in computer vision. Tekn. Lic. thesis, Linkoping University, Dep. Electrical Eng., SE-581 83 (2001)

9. Marr, D., Hildreth, E.: Theory of edge detection. Proc. Royal Society of London Bulletin 204 (1979) 301-328

10. Wilson, R., Spann, M.: Finite prolate spheroidal sequences and their applications ii: Image feature description and segmentntation. IEEE-PAMI 10 (1988) 193-203

11. Freeman, W.T., Adelson, E.H.: The design and use of steerable filters. IEEE-PAMI 13 (1991) 891-906

12. Koenderink, J.J., van Doorn, A.J.: The structure of images. Biological cybernetics 50 (1984) 363-370

13. Bigun, J., Bigun, T.: Symmetry derivatives of gaussians illustrated by cross tracking. Technical Report HH-IDE-131, Halmstad University, S-30118 Halmstad (2001)

14. Danielsson, P.E., Ye, Q.Z.: Efficient detection of second-degree variations in 2d and 3d images. Visual Communication and Image Representation 12 (2001) 255-305

15. Perona, P.: Steerable-scalable kernels for edge detection and junction analysis. In Sandini, G., ed.: Proceedings of Computer Vision (ECCV '92). Volume 588 of LNCS., Berlin, Germany, Springer (1992) 3-18

16. Black, M.J., Anandan, P.: A framework for the robust estimation of optical flow. In: ICCV-93, Berlin, IEEE Computer Society press (1993) 231-236

17. Maio, D., Maltoni, D., Cappelli, R., Wayman, J., Jain, A.K.: Fvc 2000: Fingerprint verification competition. IEEE-PAMI 24 (2002) 402-412 